\title{
Economic Benefits of Urban Wastes in Urban Landscape
}

\author{
Yijie Liu ${ }^{1, a}$ and Xiaoxia Pan ${ }^{2, b^{*}}$ \\ ${ }^{1}$ Wuhan University of Science and Technology \\ ${ }^{2}$ Hebei University of Science and Technology \\ a308864991@qq.com, b1712233051@qq.com \\ *The corresponding author
}

Keywords: Economic perspective; Urban waste; Landscape renovation

\begin{abstract}
In China, the annual emissions of urban wastes are over 105 million ton, and usually most of that huge amount of wastes is storage in an open area. Thus those wastes not only influence the city landscape but also cause contamination of water and soil, which will threat urban residents' health and city development seriously. At present, it is meaningful to recycle urban wastes reasonably in urban landscape design to achieve both energy reservation and landscape design value. In this paper, the author mainly based on economic perspective, eco-sustainability, culture sustainability and esthetic value of landscape to study the reformation and renovation of landscape in Hubei Province to provide a new method for the renovation of urban waste landscape design.
\end{abstract}

\section{Introduction}

In this paper, the author based on the evaluation of economic efficiency to study the urban wastes' application in landscape design and renovation. Through the comprehensive evaluation of the result, the author aims to provide a design principle of urban waste application in urban landscape construction, improving the hidden economic and other value of urban wastes to realize the resource recycling and design more innovative and harmonious landscape.

With the development of economy and consistently construction of cities, the industry structure, social scientific structure and social culture are changing rapidly. The increasing of urban population and construction strongly influences the urban metabolism and people's living environment and quality. There emerges a phenomenon called Drosscape, which refers to the process that the rapid urbanization produces various kinds and increasing amount of waste, and as the result the city is crowded with wastes. The urbanization process consistently destroys urban culture and ecology, which leads to the seriously waste of resource and strongly damage of urban eco-landscape. Now a days, the recycle and renovation of urban wastes has become an important part of city sustainable development. If the urban wastes can be applied in landscape construction, it can improve urban environment and the resource utilization. There are practical significance and value in the study of urban wastes' application in landscape design and renovation.

\section{Renovation Design of Wastes in Urban Landscape}

Urban wastes are generated in the maintenance of the normal operation of urban system. Those wastes are lost their value and be abandoned at last. At present the treatment methods of the wastes includes end-of-pipe treatment, resource-processing mode, and recycling mode. Among them the recycling mode is widely used in urban landscape construction, which can not only reduce the amount of urban wastes, but also present a distinctive regional custom in local area. From the economic perspective, the recycling of urban wastes in landscape construction is digging out the hidden value of the wastes to build a kind of cultural landscape. From the economic perspective, the design contains art, humanity and society three important elements, which creates a unique aesthetic perception and has received high praise.

What's more, the design and renovation of urban wastes are mainly consistent of pop art, ecological design, conservative and sustainable development, thus it is suitable to reconstruct the 
landscape from the economic perspective. From the economic perspective, the designer can dig the hidden value of urban wastes based on their unique imagination and memory to create a meaningful landscape. This concept represents the importance of aesthetic, ecological and economic value and is widely applied in modern landscape design. Through the application of urban wastes in landscape design to express the concept of aestheticism is a new perspective to appreciate the artistic value. In this case, urban wastes are the representation of humorous art which is the extraction of life to build a dialogical relationship between human and nature. It leads people to focus on nature and to rethink the relationship between human and nature. The most familiar urban wastes will be built on psychological cognition to design, which are most suitable for the development of the city. The successful design will transfer people its significant meaning and the ecologic consciousness as well.

With the development of transportation and scientific technology, the landscape design is changing rapidly. At present the landscape is hardly contains local culture and history elements. However, the old memories of urban wastes are the vital characteristics, and it can recall the old customs and technology to feel the difference of living style during the past years. Thus the local culture and history contained in urban wastes is an important element in landscape design which can complete the reasonable utilization to express the theme of landscape as well as achieving the economic conservation.

\section{Construction Logic in Landscape Design}

In order to keep its excellent durability and satisfy the social function, the materials used in landscape design should be durable and beautiful. The analysis of reuse and recycling of urban wastes in renovation of landscape can be divided into several areas, including landscape sites, landscape ornaments, decorative materials, and topographic shaping, etc. The construction of waste materials combines the traditional technique and the industrial production mode to create a new construction logic. In the landscape design, the designer selects the most suitable materials in many recycled materials to fully express its artistic expression and function and show its beauty. It is a long-term practical process to design the construction logic, meanwhile it is a process to consistently develop and improve in urban wastes' artistic expressions in landscapes.

The emergence of wastes is the result of the loss or weakness in their original function. However, with the time passed, they have accumulated historical information. From the economic perspective, the application of wastes is decreasing, but in landscape design, the wastes have the unique local customs and characteristics. In the section of landscape construction logic and design, it is important to grasp the essence of the materials including its nature and feelings to design a most suitable construction method. Thus the construction logic shall fully consider the selection of the materials and to accomplish the improvement of the materials to build reasonably in renovation of the landscape. According to different expressions like efficiency, ecology or aesthetics, the construction logic shall consider different starting points. From another perspective, the application of wastes should offer interest for people, which means the dramatic effect after the landscape combines time and space together. The wastes and the traditional landscape materials have different color and quality, which become a sharp contrast. In this situation, the wastes is a kind of signal which refers to the special construction logic that combinations of waste construction mode and the industrial construction mode. The waste may increase the artistic culture and inspiration of space to enable the designer perfectly complete the innovation of construction logic and truly express the essence and personality of waste landscape. In the wastes landscape design, it is vital important to realize the maximization of the value in the construction logic.

The crucial point in landscape design regeneration is the application of the wastes' self-value. The value of wastes are the prerequisite of landscape construction, and the value is connected with the level of the wastes. The purpose of conservative landscape construction lies in reasonable application of waste materials and natural resources, and to achieve the optimal effect of conservative landscape construction. Thus substituting wastes for original materials in landscape regeneration and innovation is the current design concept from the economic perspective. This design concept can provide double effects. One of them is in cost saving. The regeneration of wastes in landscape can save the material 
cost effectively and maximize the waste value and economic benefits. The other effect is in historical value. In the contemporary industrial construction, the landscape nearly loses its local memory value and hardly awakes the collective memory. More designers focus more on visual shock than on cultural value and spiritual expression. The application of wastes in landscape design can combine the artistic and scientific value, and comprehensively bring dual effects in spiritual and material perspective, to awake deep consciousness in human minds and bring maximum multidimensional value of the wastes.

\section{Regeneration and Updating Practice in Landscape from Economic Perspective}

One of the important elements that the designer shall consider is the scale of wastes in landscape design. It is important to select the correct wastes or facilities that conforms to the local area environment. The main urban wastes contains both large scale and small scale of wastes. The large scale wastes can be applied as an overall unit to design and play a leader role in landscape space. It can satisfy both time and space requirements to create different effects. On the other hand, the small scale wastes can be applied as an ornament. It includes building components and mechanical wastes which has little legibility in waste landscape element and environments. Thus it has the flexibility to reproduce textured patterns to complete the details processing when its texture is not suitable enough.

The more cultural connotation the objects has, the more valuable it will become. As the wastes are used to be the indispensable symbol in history, they shall be protected as a kind of culture heritage. The waste signs has their unique artistic form and feature of the original sites. According to the different color, material and scale of the wastes, it is rather suitable to apply them in different regional features, which can construct particular artistic signal and manifest the original local landscape features and charm. In this article, it mainly analyzes the construction and application of wastes landscape in Wuhan, Hubei Province. The landscape construction fully considers the features of wastes to build wastes landscape. The landscape design is the reflection of the appearance and culture in Wuhan, Hubei Province. In addition, it shows the resource advantages and has an important meaning in the improvement of wastes' value.

Through the technological renovation to regenerate wastes into new materials is the concept in this landscape design. As shown in Figure 5, the designer selects the waste composite material in a flexible space to renovate the original structure composed with waste residues. Meanwhile, the paving materials are made of rubbles from the demolishing of waste buildings. Its surface is made of rubber to recreate a thrilling public place, which provides more entertainment and pleasure for people.

\section{Acknowledgements}

The project is supported by the heavy industry landscape development based on market and economy in the program of Department of Construction. (No. K201183)

\section{References}

[1] Waldheim Charles. The Landscape Urbanism Reader (Hailong Liu, Dongyun Liu, Lu Sun, Trans) [M]. Beijing: China Architecture \& Building Press, 2011.11-14.

[2] Hanming, Duan. Introduction to Urban Aesthetics and Landscape Design [M]. Beijing: Higher Education Press, 2008. 25-26.

[3] Rui Yang, Landscape Urbanism Application in Brownfield Regeneration [J]. Modern Urban Research, 2008, (10):71-76.

[4] Jun Zhai, Scape-city Based on Landscape Urbanism [J]. Architectural Theory, 2010, (11):6-11.

[5] Xianming Tu, Luohu Wenjin Garden Plaza, Shenzhen [J], Landscape Architecture China, 2012, (24):80-82. 
[6] Li Dong, Xiangrong Wang. Low Intervention, Low Consumption, Low Conservation, and Low Emission---Research on Low-cost Landscape Architecture [J]. Chinese Landscape Architecture, 2013.5.

[7] Liwen, Gu. The Application of Waste Materials in Road Engineering [J]. China \& Foreign Highway, 2002, 2:53-55.

[8] Zhiyan Chen, Refelctions on Conservation Ecological Landscape Construction [J]. Junyi Huang. Journal of Heilongjiang Vocational Institute of Ecological Engineering, 2009, (2):15-16.

[9] Tongyu Li, Guangtian Zou. Study on Modes of Landscape Design with Waste Materials [J]. Building Science, 2011, (12):6-15. 